\title{
Parapharyngeal space lipoma: a case report
}

\author{
Herbert SH Lee *, MB, ChB (CUHK), CM Ngai, MB, BS, FHKAM (Otorhinolaryngology)
}

Department of Otorhinolaryngology, Head and Neck Surgery, Yan Chai Hospital, Kowloon West Cluster, Hong Kong

* Corresponding author: herbertshlee@link.cuhk.edu.hk

Hong Kong Med J 2020;26:438-40

https://doi.org/10.12809/hkmj198214

\section{Introduction}

Parapharyngeal space (PPS) tumours account for only around $0.5 \%$ of tumours in the head and neck region. ${ }^{1}$ Most are benign in nature and salivary in origin. ${ }^{2}$ Lipomas in the PPS are rare, despite being the most common benign mesenchymal head and neck tumour., ${ }^{3,4}$ Other lesions that may be encountered in this area include neurogenic tumours, chemodectomas, branchial cysts, and metastatic lesions.

\section{Case report}

A middle-aged woman first presented to the outpatient clinic in April 2019 with a 3-year history of left painless neck mass, with gradual growth but no other symptoms. Physical examination revealed a soft neck mass of around 4 to $5 \mathrm{~cm}$ in diameter over the left level III region. Other ear, nose and throat examinations, including flexible laryngoscopy, were unremarkable.

Initial fine-needle aspiration cytology (FNAC) was inadequate for diagnosis. A second FNAC revealed sparse adipose tissue. Ultrasound showed a $2.68 \times 1.52 \times 2.41 \mathrm{~cm}$ deeply located hypoechoic lesion on the left submandibular region, with internal striations resembling those of subcutaneous fat. No vascularity was detected on colour Doppler scan. Oval lymph nodes with preserved fatty hilum were seen at bilateral upper cervical regions. They all had a sub-centimetre short axis, likely reactive in nature. The overall sonographic impression was of lipoma. Magnetic resonance imaging (MRI) revealed a $3.6 \times 4.9 \times 9.2 \mathrm{~cm}$ (anteroposterior $\times$ transverse $\times$ craniocaudal planes) well-circumscribed, lobulated mass in the left PPS. The lesion was of fat signal intensity, with faint fluffy internal enhancing septation but no internal enhancing solid nodule seen, nor frank invasion into the surrounding structures (Fig 1a). The lesion extended from the level of $\mathrm{C} 1$ to $\mathrm{C7}$ (Fig 1b), bulging medially onto the left side of the oropharynx and displacing the left carotid space content posterolaterally (Fig 1c). The MRI findings were suggestive of a lipomatous lesion. The overall clinical picture was compatible with a parapharyngeal lipoma. Although parapharyngeal lipoma is a benign lesion, gradual growth and consequent mass effects can occur. As the patient was only middle-aged, complications such as dysphagia, shortness of breath, and obstructive sleep apnoea were possible if the tumour was allowed to continue growing. After explaining to the patient the clinical, FNAC and imaging findings, as well as potential complications arising from the tumour, she
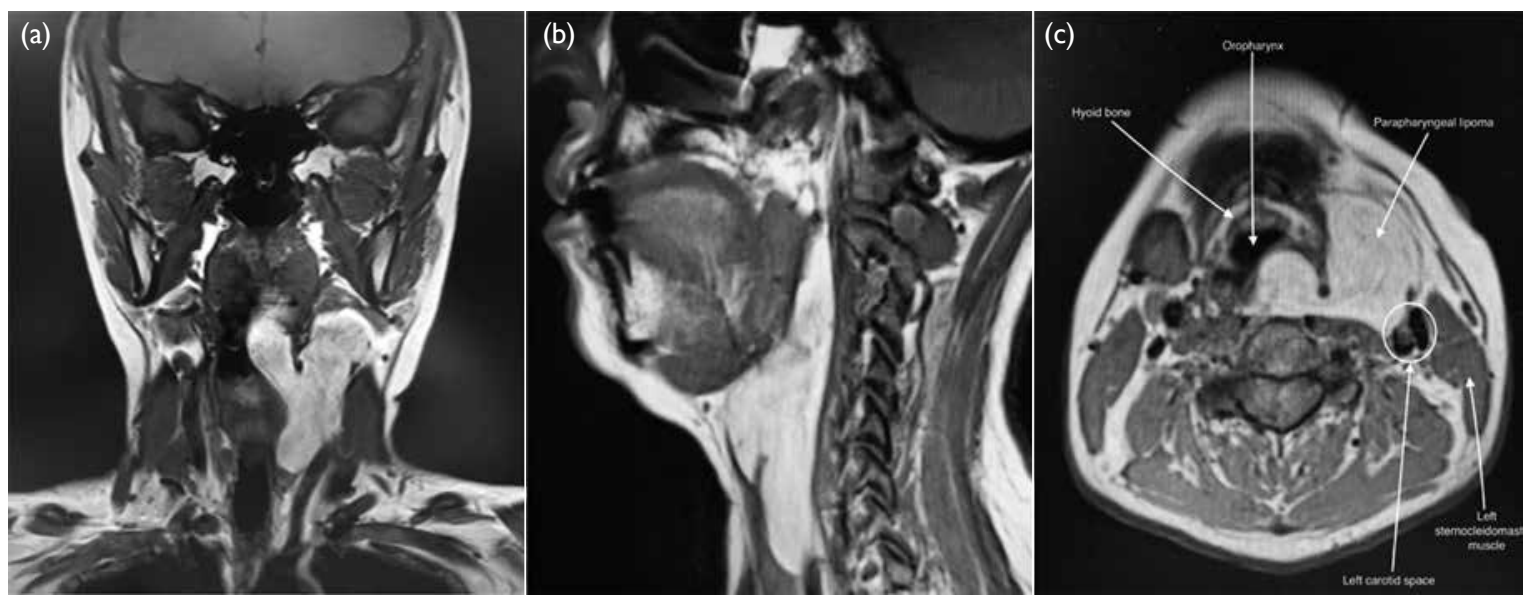

FIG I. (a) Coronal magnetic resonance image showing a mass lesion of fat signal intensity in the left parapharyngeal space. (b) Sagittal magnetic resonance image showing the extent of the lesion from $\mathrm{Cl}$ to $\mathrm{C7}$. (c) Axial magnetic resonance image showing displacement of the oropharynx and left carotid space by the lesion 
was keen to undergo surgical excision.

During out-patient preoperative assessment in July 2019, the patient complained of recent dull vague pain in the throat. Physical examination revealed the hyoid bone to be deviated to the right. Subsequent flexible laryngoscopy demonstrated a swelling on the left lateral oropharyngeal wall.

Surgery under general anaesthesia was performed in August 2019 and the sternocleidomastoid muscle was dissected from the lipoma. The lipoma was subsequently dissected from the internal jugular vein and the carotid artery and completely excised (Fig 2). Haemostasis was achieved and a drain inserted prior to skin closure. The entire operation took about 2 hours with intraoperative blood loss of around $50 \mathrm{~mL}$. The patient was discharged 5 days later with no complications noted.

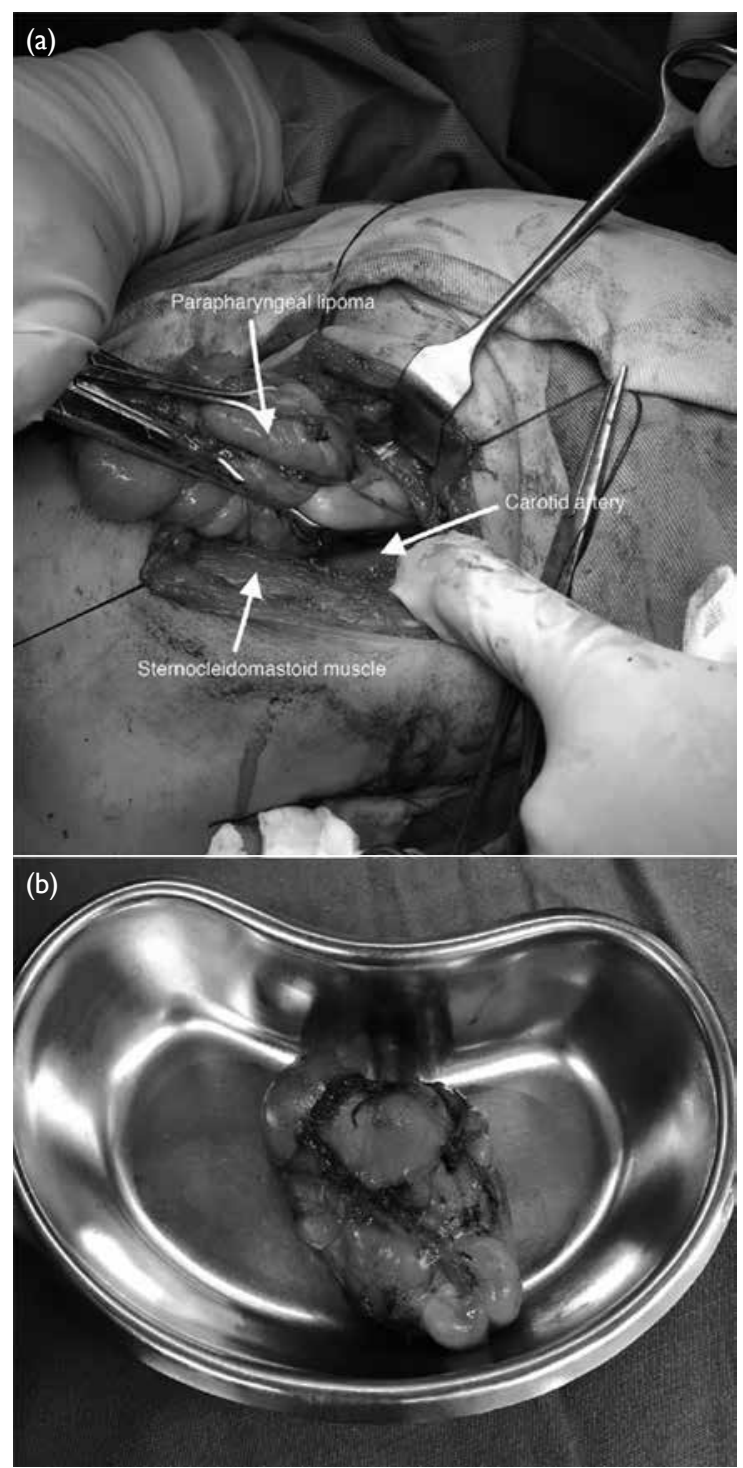

FIG 2. (a) Intra-operative photograph showing the lipoma. (b) Photograph of the excised parapharyngeal lipoma
At postoperative follow-up 9 days later the patient was clinically well with no active complaints. She was tolerating an oral diet and the wound had healed well. Flexible laryngoscopy showed no more oropharyngeal wall swelling. Pathology results confirmed that the left parapharyngeal lesion was a lipoma and the excised left level III lymph node had no evidence of malignancy.

\section{Discussion}

The PPS is anatomically described as an inverted pyramid with the apex and the base at the greater cornu of the hyoid and the skull base, respectively. The posterior boundary is the vertebral column and paravertebral muscles. Anteriorly, it is limited by the junction of pterygoid fascia to the buccinator muscle fascia, the pterygomandibular raphe, and the submandibular gland. The medial boundary is formed by the superior constrictor muscle and the tonsillar fossa. The lateral boundary is formed by the medial pterygoid muscle, the ramus of mandible, parotid gland, and the posterior belly of the digastric muscle. The PPS can be subdivided into pre-styloid and post-styloid compartments by the styloid process. The post-styloid compartment consists of several vital structures including the 9th to 11th cranial nerves, internal carotid artery, internal jugular vein, and cervical sympathetic trunk. The pre-styloid compartment may contain the deep lobe of the parotid gland or accessory salivary tissues, as well as lymph nodes. A lipoma found in the pre-styloid compartment of the PPS is indeed very rare.

A lipoma is an encapsulated, benign, subcutaneous, and submucosal tumour composed of mature adipose tissue cells. Most PPS lipomas grow insidiously and cause symptoms only when exerting mass effects as seen in any other benign tumours, for example, dysphagia, shortness of breath, and obstructive sleep apnoea. Occasionally, due to obstruction of the Eustachian tube by the tumour, otitis media with effusion and conductive hearing loss may occur. Hoarseness and tongue muscle weakness resulting from compression of the lower cranial nerves, as well as Horner's syndrome and trismus owing to involvement of the cervical sympathetic trunk and medial pterygoid respectively are all suggestive of malignancy.

Diagnostic FNAC is often technically difficult as the PPS lipoma is deep-seated. Most of the time, imaging such as computed tomography (CT) and MRI has significant diagnostic significance. On computed tomography scan, a lipoma is revealed as a homogenous and hypodense mass with no enhancement. In contrast, MRI scan is the most ideal imaging modality due to its excellent delineation of soft tissue and multiplanar capability. Lipomas appear as hyperintense on T1- and T2-weighted sequences with internal septations. T1-weighted 
sequences with fat suppression demonstrate even more obvious contrast with the surrounding soft tissues.

Surgical excision is the treatment of choice. The surgical approach depends on tumour size, location, and relationship to major vessels. A transcervical approach is most widely applied and is particularly suitable for smaller PPS tumours. Some literature suggests a transcervical approach for tumours as large as $8 \mathrm{~cm}, 5,6$ but there is no consensus on the cut-off size. Our case demonstrates that the transcervical approach can achieve good exposure for a parapharyngeal tumour extending from level $\mathrm{C} 1$ to $\mathrm{C} 7,9.2 \mathrm{~cm}$ at its greatest dimension. Advantages of a transcervical approach include adequate exposure of vital structures and a lower risk of damage to the facial nerves. A transcervical approach is combined with a transmandibular approach when dealing with larger lesions involving the skull base and the aforementioned lower cranial nerves for better exposure, while an infratemporal approach is used when access to the lateral part of the skull base is necessary. A transoral approach, which was used in the past, is now out of favour as the exposure offered by this route is very poor. There is also a higher risk of vascular and neural injury that makes this approach unsafe.

\section{Conclusion}

Parapharyngeal space lipomas are rarely seen in our daily practice but deserve more of our attention since they are easily missed in the early stages and seen as a non-specific neck mass as in this case. Magnetic resonance imaging scan is useful not only in terms of diagnostic superiority, but also for preoperative planning. A transcervical approach is most commonly adopted for PPS tumours as it is associated with lower surgical risk by providing excellent exposure of vital neurovascular structures.

\section{Author contributions}

Concept or design: CM Ngai.

Acquisition of data: HSH Lee.

Analysis or interpretation of data: HSH Lee.

Drafting of the manuscript: HSH Lee.

Critical revision of the manuscript for important intellectual content: CM Ngai.

All authors had full access to the data, contributed to the study, approved the final version for publication, and take responsibility for its accuracy and integrity.

\section{Conflicts of interest}

All authors have disclosed no conflicts of interest.

\section{Funding/support}

This case report received no specific grant from any funding agency in the public, commercial, or not-for-profit sectors.

\section{Ethics approval}

The study was conducted in accordance with guidelines by the Kowloon West Cluster ethics committee. Informed consent was obtained from the patient.

\section{References}

1. Carrau RL, Johnson JT, Myers EN. Management of tumors of the parapharyngeal space. Oncology (Williston Park) 1997;11:633-40.

2. Batsakis JG, Sneige N. Parapharyngeal and retropharyngeal space diseases. Ann Otol Rhinol Laryngol 1989;98:320-1.

3. Abdullah BJ, Liam CK, Kaur H, Mathew KM. Parapharyngeal space lipoma causing sleep apnoea. Br J Radiol 1997;70:1063-5.

4. Ulku $\mathrm{CH}$, Uyar Y. Parapharyngeal lipoma extending to skull base: a case report and review of the literature. Skull Base 2004;14:121-5.

5. Smith JC, Snyderman CH, Kassam AB. Giant parapharyngeal space lipoma: case report and surgical approach. Skull Base 2002;12:215-20.

6. Carrau RL, Myers EN, Johnson JT. Management of tumors arising in the parapharyngeal space. Laryngoscope 1990;100:583-9. 